\title{
Critical evaluation of transcription factor Atf 2 as a candidate modulator of alcohol preference in mouse and human populations
}

\author{
L.S. Wang ${ }^{1}$, Y. Jiao ${ }^{1}$, Y. Huang ${ }^{1}$, X.Y. Liu ${ }^{1}$, G. Gibson ${ }^{1}$, B. Bennett ${ }^{2}$, \\ K.M. Hamre ${ }^{3}$, D.W. Li ${ }^{4}$, H.Y. Zhao ${ }^{5,6}$, J. Gelernter, ${ }^{4,6,7}$, H.R. Kranzler, ${ }^{9,10}$, \\ L.A. Farrer ${ }^{11}$, L. Lu ${ }^{3}$, Y.J. Wang ${ }^{12}$ and W.K. Gu ${ }^{1}$
}

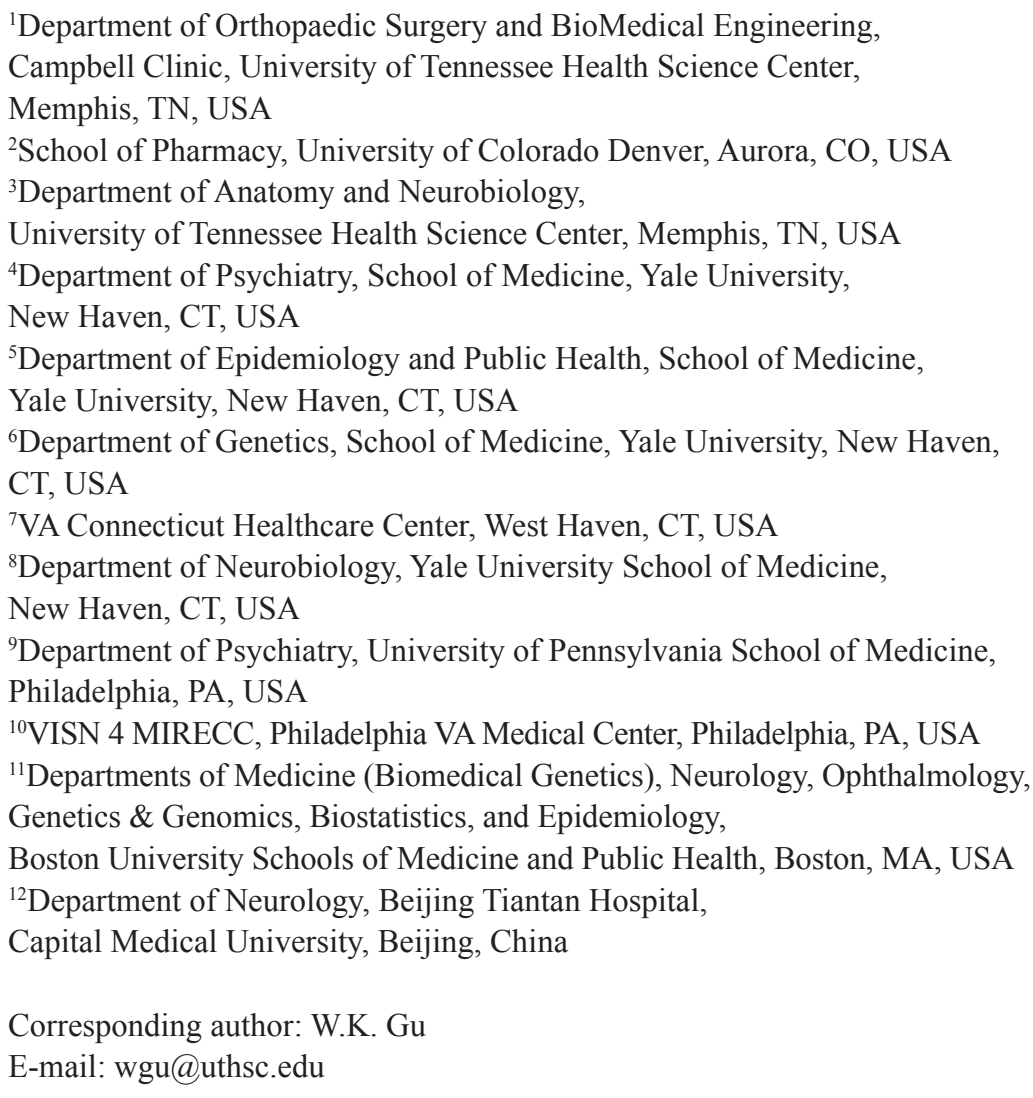


Genet. Mol. Res. 12 (4): 5992-6005 (2013)

Received February 27, 2013

Accepted September 15, 2013

Published November 26, 2013

DOI http://dx.doi.org/10.4238/2013.November.26.9

ABSTRACT. In prior work, congenic strains carrying the DBA/2Igb (D2) region of chromosome $2(\mathrm{Chr} 2)$ for alcohol preference were bred onto a $\mathrm{C} 57 \mathrm{BL} / 6 \mathrm{Ibg}$ (B6) background and as predicted were found to reduce voluntary consumption. Subsequently, interval-specific congenic recombinant strains (ISCRS) were generated and also tested. These ISCRS strains reduced the quantitative trait loci (QTL) interval to a comparatively small $3.4 \mathrm{Mb}$ region. Here, we have exploited an integrative approach using both murine and human populations to critically evaluate candidate genes within this region. First, we used bioinformatics tools to search for genes relevant to alcohol preference within the QTL region. Second, we searched for single nucleotide polymorphisms (SNPs) within exons of every gene in this region. Third, we conducted follow-up microarray analyses to identify differentially expressed genes between the B6 and ISCRS strains in mice from each group. Fourth, we analyzed correlations between the expression level of candidate genes and phenotypes of alcohol preference in a large family of BXD recombinant inbred strains derived from B6 and D2. Finally, we evaluated SNP segregation in both BXD mouse strains and in humans who were heavy alcohol drinkers or non-drinkers. Among several potential candidate genes in this region, we identified activating transcription factor 2 (Atf2) as the most plausible gene that would influence alcohol preference. However, the candidacy of Atf 2 was only weakly supported when we used a genetic network approach and by focused reanalysis of genome-wide association study data from European-American and African-American populations. Thus, we cannot conclude that Atf2 plays a role in the regulation of the QTL of mouse Chr2.

Key words: Alcohol preference; Atf2; Candidate; QTL; Gene expression

\section{INTRODUCTION}

Many quantitative trait loci (QTL) influencing responses to alcohol have been mapped using a variety of mouse models and genetic approaches. With important exceptions, the gene variants that underlie these multiple QTLs (QTG) are still unknown. In a previous work, Bennett et al. (2002) created congenic strains carrying the DBA/2IBG (D2) region for alcohol preference on chromosome 2 (Chr2) on a C57BL/6IBG (B6) background. Subsequently, interval-specific congenic recombinant (ISCR) strains in which the full D2 QTL region was broken into smaller, partially overlapping regions of introgression, were generated and tested. QTGs are genes with unknown function or whose functional relationship to the quantitative phenotype is not immediately apparent. For this reason, identifying QTG(s) in a candidate interval must include every gene in that region. In the past, applying this strategy 
to biomedical research was limited by the availability of genomic data and technology. Now, with the completion of the human and mouse genomic sequence and the development of high throughput technology for polymorphic analysis and for gene expression profiling, we can use a genomic, bioinformatics-driven approach to identify QTGs. In conjunction with the availability of the mouse genome sequence, there have been other relevant developments. First, we can rapidly identify almost every single nucleotide polymorphism (SNP) of a given population by high throughput sequencing. This development is significant for identifying QTGs because a QTL is largely considered to be regulated by polymorphisms, and a QTL region typically contains many genes in a large genomic region. Second, a gene expression profile of the whole genome can be easily generated by gene microarray technology. Furthermore, data from animal models can be double checked in a study of humans. We report here the initial analysis of genes within a defined QTL region by using a combination of bioinformatics, gene expression profiling, SNP analysis, and transcriptome pathway analyses.

\section{MATERIAL AND METHODS}

\section{Animals}

Animals were produced at the Institute for Behavioral Genetics at the University of Colorado, Boulder (UCB). Accordingly, animals carrying the full congenic region, as well as one ISCR strain with the smallest interval conferring the D2 phenotype (strain 21), were used. Tissues were collected at UCB and shipped to the University of Tennessee Health Science Center (UTHSC) at Memphis. Experimental procedures for this study were approved by the Institutional Animal Care and Use Committee at UCB.

\section{DNA and RNA extraction}

Genomic DNA was extracted from livers of both wild type and ISCR mice by using a Qiagen DNA kit (Valencia, CA, USA) and following manufacturer instructions. After determining the quality and quantity of the DNA in an Eppendorf photometer (Eppendorf Scientific Inc., Westbury, NY, USA), DNA was used for sequencing and SNP screening. RNA was extracted from different parts of the brain by using a Trizol reagent (Invitrogen, Carlsbad, CA, USA). Total RNA was purified using the RNeasy MinElute Cleanup kit (Qiagen). RNA quality and integrity were checked using an Agilent Bioanalyzer (Davis, CA, USA). DNA and RNA from three replicates of ISCR strains and wild type controls were extracted for each experiment.

\section{Microarray experiment}

Microarray analysis was conducted using RNA from brain parts of three ISCR mice and three wild type mice. A starting amount of $200 \mathrm{ng}$ high-quality total RNA, with an RNA integrity score of more than 7, was used to generate cDNA and cRNA with the Illumina TotalPrep RNA Amplification kit (Ambion, Foster City, CA, USA). For each of six individual samples, $1.5 \mu \mathrm{g}$ cRNA sample was hybridized overnight to the Mouse-6 v1B BeadChip in a multiple-step procedure according to manufacturer instructions; the chips were then washed, dried, and scanned on the BeadArray Reader (Illumina, San Diego, CA, USA). The raw data were generated using BeadStudio 2.3.41 (Illumina,). 
Data analysis followed our previous procedure (Jiao et al., 2011). Briefly, raw data were normalized by the quantile method with BeadStudio software. Upon detection of $\mathrm{P}<$ 0.05 and fold-change $(\leq 2)$ filtering, statistical analysis was performed using EDGE software to generate differentially expressed genes, which were subject to hierarchical and functional clustering using Cluster/TreeView and bioinformatics DAVID tools (http://david.abcc.ncifcrf. gov), respectively. To identify differentially expressed genes between different groups, a cutoff value of 2-fold changes was chosen, which was based on most published studies (Jeffrey et al., 2002; Yan et al., 2007; Sharov et al., 2008).

\section{Bioinformatics evaluation of candidates}

Genome screening was performed using a bioinformatics approach to identify genes regulating alcohol preference in the mouse genome. A gene was considered to be an alcoholismassociated gene if it was associated with alcoholism or alcohol preference in at least one of the following studies: 1) functional studies such as knockouts, transgenics, mutagenesis, or RNA interference; 2) association studies; or 3) clinical studies. We searched all chromosomes and QTL in the Ensembl database (Release 48) (http://www.ensembl.org/index.html). We then searched PubMed (http://www.ensembl.org/index.html) and OMIM (Online Mendelian Inheritance in Man; http://www.ncbi.nlm.nih.gov/sites/entrez) to obtain a preliminary set of candidate genes associated with alcohol preference. The search terms were the gene symbol and alcohol preference. We completed the search with PGMapper, a software package developed by our laboratory and available at http://www.genediscovery.org/pgmapper/index.jsp (Xiong et al., 2008).

\section{SNP searching}

Known SNPs in the exons of genes within the QTL region were examined in available SNP databases, such as the Roche SNP database, MGI mouse SNP database at http://www. informatics.jax.org/javawi2/servlet/WIFetch?page=snpQF, and NCBI SNP database at http:// www.ncbi.nlm.nih.gov/SNP/ and the Variant Browser of GeneNetwork (http://www.genenetwork.org/webqtl/main.py?FormID=snpBrowser).

\section{Genomic sequencing}

Sequencing and polymorphisms between B6 and D2 strains were obtained from GeneNetwork at UTHSC: http://ucscbrowser.genenetwork.org/cgi-bin/hgGateway. The D2 sequence was generated in-house at UTHSC to a depth of $100 \mathrm{X}$ and then combined with $23 \mathrm{X}$ coverage sequence from the Sanger Institute. We used the GeneNetwork website (http://ucscbrowser.genenetwork.org) to obtain the phenotypes of alcohol preference and to search SNPs and nucleotide insertions and deletions between B6 and D2 strains. Polymorphisms were confirmed by amplifying DNA fragments of polymorphic sites and re-sequencing PCR fragments using conventional Sanger protocols at UTHSC. Confirmed polymorphisms were screened for their distribution among BXD strains.

\section{Gene expression profiles in BXD family}

Gene expression patterns of candidate genes were obtained from GeneNetwork. Ex- 
pression of each candidate gene was examined in multiple BXD family members (more than 30 strains in each tissue) in three tissues: whole brain, hippocampus, and cerebellum; each of those tissues has been used for the study of gene expression (Kamens et al., 2009; Fitzgerald et al., 2011; Parsons et al., 2012)

\section{Association of candidate genes in human populations}

This part of the research was conducted at Yale University. A total of 5,685 subjects were recruited at the Yale University School of Medicine, University of Connecticut Health Center, Medical University of South Carolina, University of Pennsylvania School of Medicine, and McLean Hospital. Subjects were selected because screening suggested that they had cocaine, opioid, or alcohol dependence, or were normal controls. All subjects were interviewed using an e-version of the Semi-Structured Assessment for Drug Dependence and Alcoholism (SSADDA) (Gelernter et al., 2005; Pierucci-Lagha et al., 2007) and diagnoses were made on the basis of Diagnostic and Statistical Manual of Mental Disorders-IV (DSM-IV) criteria (American Psychiatric Association, 1994). Subjects gave informed consent, as approved by the institutional review boards at each site. The subjects were genotyped using the Illumina HumanOmni1-Quad array (Illumina) at either the Yale Center for Genome Analysis or Center for Inherited Disease Research (CIDR). For quality controls, samples with poor genotyping performance were replaced with substituted DNA. Samples with discrepant gender information, unusual X and $\mathrm{Y}$ chromosome patterns or unexpected duplicated DNA were removed. Furthermore, samples that were duplicated, from nonAfrican-American or non-European-American populations, related, or of unknown phenotype were excluded (unpublished manuscript). All 38 common SNPs (minor allele frequency $>0.05$ ) in the Atf 2 gene were analyzed, followed by additional imputted genotypes of 416 common SNPs in the gene region. The allelic analysis was carried out using a chi-square association test. The combined analyses of the African and European American samples were performed via metaanalysis. Additional genotypes were imputted using IMPUTE2 (Howie et al. 2009). Bonferroni correction was applied for multiple comparisons.

\section{RESULTS}

\section{Initial identification of candidate genes in the QTL region from interval-specific congenic recombinant strains}

The QTL was fine-mapped to a $3.4 \mathrm{Mb}$ region between D2Mit56 (70.4 Mb) and D2Mit10 ( $73.8 \mathrm{Mb}$ on the Mm9 genome assembly). The genome sequence in this region is complete, and gene annotations are generally of high quality.

By searching the mouse genome database using PGMapper (Xiong et al., 2008), we identified a total of 68 genes and other genetic elements ( $\underline{\text { Table S1) }}$ in the region between D2Mit56 and D2Mit10.

Among these genes, we found five with potential function in alcohol preference (Table 1). Atf 2 (activating transcription factor 2) is involved in the mitogen-activated protein kinase (MEKK1) $M K K 7 / J N K 2$ ) pathway, which has been shown to be altered in alcoholism (Chen et al., 1998; Roberto et al., 2003). Atp5g3 [ATP synthase, $\mathrm{H}^{+}$transporting, mitochondrial F0 complex, subunit c (subunit 9), isoform 3] has been linked to chronic ethanol consumption (Li et al., 2001). Pdk1 (py- 
ruvate dehydrogenase kinase, isoenzyme 1) has been linked to chronic ethanol consumption (Yu et al., 2011). $S p 3$ and $S p 9$ are both trans-acting transcription factors, which recognize target sequences and interact with other transcription factors to regulate the expression of other genes. Because their regulatory functions have not been clearly reported, they cannot be ruled out as candidates.

\begin{tabular}{|c|c|c|c|c|c|}
\hline Ensembl accession & Symbol & Full name & No. of SNPs & Insertions & Deletions* \\
\hline ENSMUSG00000027104 & Atf 2 & Activating transcription factor 2 & 3 & & \\
\hline ENSMUSG00000027109 & Sp3 & Trans-acting transcription factor 3 & $1(\mathrm{G} / \mathrm{A})$ & & -AT (72784944) \\
\hline ENSMUSG00000006494 & Pdkl & Pyruvate Dehydrogenase kinase, Isoenzyme 1 & & & $-\mathrm{T}(71718212)$ \\
\hline ENSMUSG00000027107 & Chrnal & Cholinergic receptor nicotinic, Alphapolypeptide 1 & & & \\
\hline ENSMUSG00000018770 & Atp5g3 & $\begin{array}{l}\text { ATP synthase, } H+\text { transporting, mitochondrial F0 } \\
\text { complex, subunit } C \text {, (subunit 9), isoform } 3\end{array}$ & & & \\
\hline
\end{tabular}

*The numbers in the parentheses are the nucleotide position of deletions within QTL on mouse chromosome 2. (-) Denotes the deletions of the nucleotides.

\section{SNP summary data for candidate genes}

Atf2 is located within the genomic region of the QTL (Table 1). We obtained SNP information from MGI as well as from the genome sequence information at GeneNetwork. Our initial data identified two SNPs: an A/C SNP at position Chr2 73.673213 Mb (Assembly Mm9) and a C/T SNP at position Chr2 73.706059. Mb. In addition, there was a nucleotide "G" deletion at position $\mathrm{Chr} 273.726407 \mathrm{Mb}$ in the $\mathrm{D} 2$ strain. When we sequenced this region, we confirmed the SNP A/C at 73.673213. However, the putative SNP at 73.706059 or a deletion at 73.726407 $\mathrm{Mb}$ was not confirmed.

Atp5g3 is located within the genomic region of the QTL (Table 1). However, according to the MGI site, this gene does not contain a SNP among the mouse strains, even when examining $2000 \mathrm{bp}$ upstream or downstream. No polymorphisms were confirmed by the sequencing data at UTHSC.

Pdkl is located within the genomic region of the QTL (Table 1). On the basis of both MGI and sequencing data at UTHSC, we confirmed that the gene contains 44 SNPs, but none differs between the B6 and D2 strains, including 2000 bp upstream or downstream.

According to the MGI database, Sp 3 has no SNPs in mouse strains. However, UTHSC sequence data indicated a polymorphism at position $\mathrm{Chr} 272.792729 \mathrm{Mb}$.

$S p 9$ has three SNPs, but they are the same genotype in the B6 and D2 strains.

Although the absence of a polymorphism does not completely rule out the candidacy of Atp5g3, Pdk1, and Sp9, those results warranted focus on Atf2 and $S p 3$.

\section{Gene expression profiles of candidate genes in B6 and ISCR strains}

Whole-genome expression levels of more than 30,000 genes in whole brain from B6 and the full congenic strain (G), in hippocampus from B6 and G, and in cerebellum from B6 and ISCR 21 were analyzed using microarray. Each experiment was conducted using three mice from each strain.

At the whole-brain level, we found 135 genes that were downregulated and 114 that were upregulated in the ISCR stains (Table S2). We then examined the expression levels of 
Atf2, Atp5g3, and Pdk1. The data suggested that the expression level of Atf 2 in the G strain was significantly downregulated $(\mathrm{P}=0.035)$ (Figure 1A) compared to that in $\mathrm{B} 6$. The expression levels of Pdk1 (Figure 1A) and Atp5g3 (Figure 1B) showed differences between B6 and ISCR strains, but not at a significant level. There was no difference in expression levels of sp3 and sp9 genes between B6 and G strains (Figure 1A).

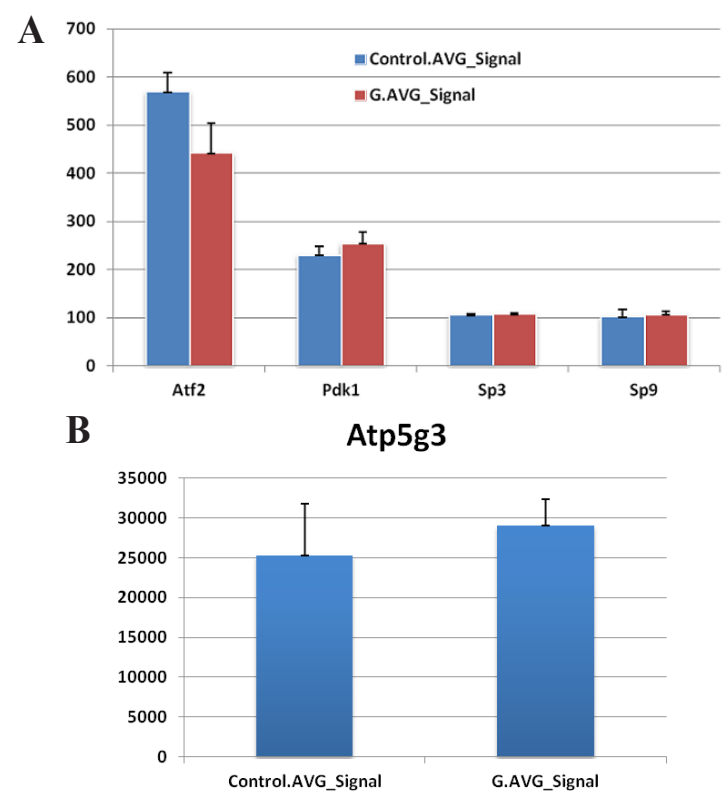

Figure 1. Expression levels of Atf2, Atp5g3, and Pdk1 in whole brain between C57BL/6J and ISCRS strains. Y axis indicates the relative strength of the signal of the gene expression, normalized among samples. A. Expression level of Atf 2 in the G strain was significantly downregulated compared to that in B6. There is a difference but not at a significant level, in expression level of $P d k 1$ between B6 and ISCR strains. There is no difference in expression levels of $S P 3$ and $S P 9$ genes between B6 and G strains B. There is a significant difference in expression Atp5g3 between B6 and ISCR strains.

The gene expression level was further tested using microarray data from hippocampus between B6 and G strains. Atf2 expression level in the G strain was lower than that in the B6 strain (Figure 2A), Similarly, Atp5g3 showed an increase in the G strain. The expression levels of the other three candidates, however, showed no difference between the B6 and G strains (Figure 2B).

A third microarray experiment was conducted using cerebellum from B6 and ISCR 21. Expression level of Atf 2 in the ISCR strain was again downregulated (Figure 3A). Atp5g3 expression level was significantly increased in the $\mathrm{G}$ strain (Figure 3B). The expression levels of the other three candidates were similar to those in the B6 strain.

According to the data that there is no difference in expression profiles of $S p 3$ and $S p 9$ between B6 and G strains, there were no nucleotide polymorphisms between the two strains. Although there was difference in the gene expression level of Atp5g3 between B6 and G strains, there was no polymorphism between the two strains. Atf 2 was then considered as the most likely candidate gene showing both differential expression level and polymorphisms that were the focus of our investigation. 


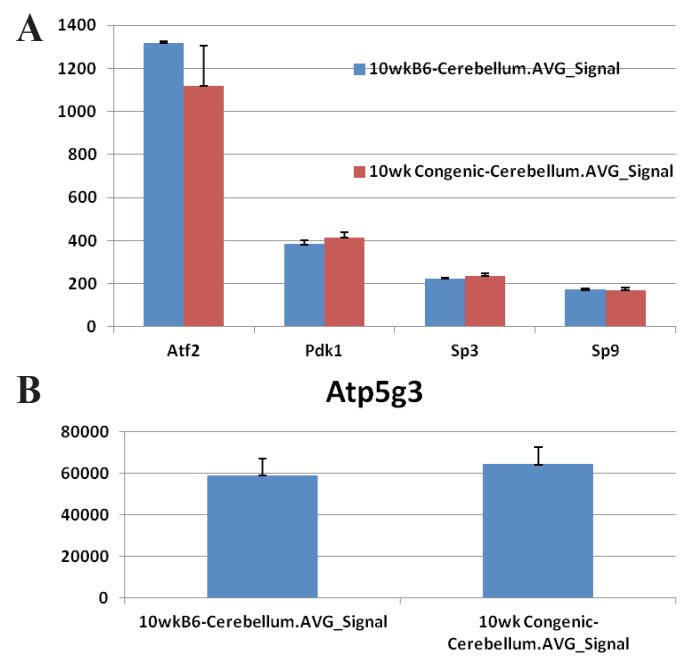

Figure 2. Gene expression level in hippocampus between B6 and G strains. Y axis indicates the relative strength of the signal of the gene expression, normalized among samples. A. Atf2 expression level in the ISCR strain was lower than that in the B6 strain. The expression level of Pdk1, SP3 and SP9 shows no significant difference between B6 and ISCR strains. B. Atp $5 \mathrm{~g} 3$ showed an increase in the G strain.

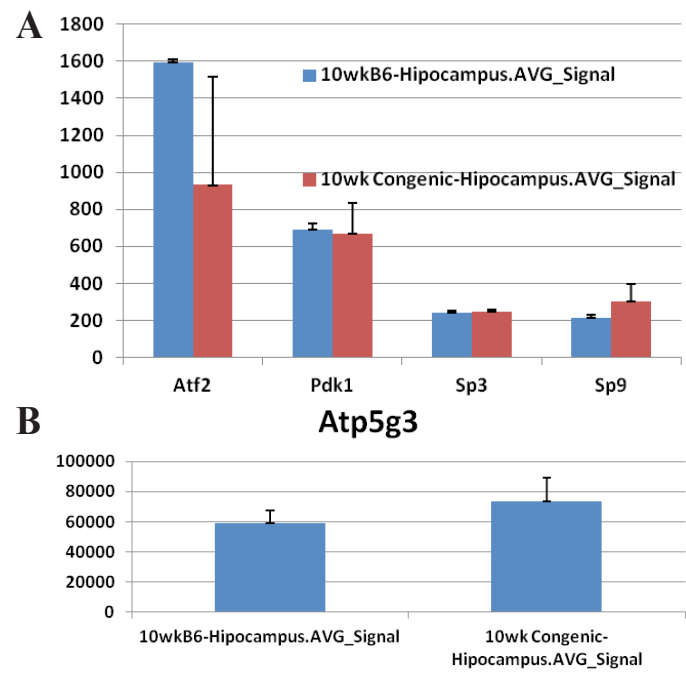

Figure 3. Gene expression level in cerebellum from B6 and ISCRS 21 strain. Y axis indicates the relative strength of the signal of the gene expression, normalized among samples. A. Expression level of Atf 2 in the \#21 strain was again downregulated. The expression levels of the other three candidates were similar to those in the B6 strain. B. Atp5g3 expression level was increased significantly in the ISCRS 21 strain.

\section{QTL location of alcohol preference on chromosome 2 in the BXD strain family}

Several groups have mapped QTLs for alcohol consumption using the original set of BXD strains (Gill et al., 1996; Fernandez et al., 1999; Grisel et al., 2002; Philip et al., 2010). We examined the potential correlation between expression of Atf 2 and alcohol preference 
tested using the two-bottle choice method. Because we found a differential expression of Atf2 between $\mathrm{B} 6$ and congenics in three tissues, we examined the association between expression of Atf 2 in brain, hippocampus, and cerebellum and alcohol preference in a BXD family. Expression profiles for BXD strains were generated using the Affymetrix MOE 4302.0 array.

\section{Co-expression of Atf2 in whole brain and alcohol preference}

Probe 1452116, from the distal 3'-untranslated region of Atf 2 , had a positive correlation with average consumption during a 3-week free choice period using the two-bottle choice test for males in a study by Gill et al. (1996) (Record ID 10155 in the BXD Phenotypes data set in GeneNetwork). The expression level was correlated to the data for average amount of ethanol consumed for body weight for a 2-week period (Fernandez et al., 1999) (Record ID 10135). Expression was positively related to total consumption over $24 \mathrm{~h}$ for males (Rodriguez et al., 1992) (ID 10582) (Figure 4A, B, C). Although the number of BXD strains in those three studies was relatively small, the data agreed with the microarray study of B6 and congenic strains.

\section{Co-expression of Atf2 in hippocampus and alcohol preference}

In hippocampus, probe 1452116 was positively correlated with average ethanol consumption $(\log \mathrm{g} / \mathrm{kg} / 2 \mathrm{~h}$ ) of BXD strains at 2 (Record ID 12578) and 4 weeks (Record ID 12576) from a study using two-bottle of choice and 2-h access. Figure 5A, B shows the corelationship between the expression of Atf2 and alcohol preference of BXD strains. The data are convincing as the number of strains in those analyses is relatively large (around 40 strains).

In the co-expression of Atf2 in cerebellum and alcohol preference, similar to that in whole brain, expression of Atf2 (Probe 1452116) positively correlated with the amount of average ethanol consumption in week 3 for BXD strains using a two-bottle choice, 2-h access (g/kg per $2 \mathrm{~h}$ ) (Figure 5C) (ID 12577).

Interestingly, the expression of Atf 2 was also correlated with ethanol consumption from data of 3 days after cycle 1 of chronic intermittent ethanol vapor in a recently study (Unpublished Record ID 12816 in GeneNetwork) using 2-bottle choice consumption (Figure 5D).

\section{SNP genotype of $A t f 2$ and alcohol consumption in BXD strains}

In spite of the clearly defined QTL region on Chr2 and its role in alcohol preference in the ISCR mice, potential candidate genes for this QTL, including the BXD population, have remained unidentified. One difficulty with the study of alcohol using the mouse model is that a report suggested that more than 50 mice would be required to detect a significant difference with a P value of 0.05 or less (Ruf et al., 2004). We assume that if the effect of QTL on Chr2 were large enough, we might see differences in alcohol preference between cases categorized by their SNP genotypes. However, because alcohol preference is influenced by multiple genetic and environmental factors, the difference may or may not reach the statistically significant level. On the basis of our assumption, we genotyped BXD strains at the Atf2 SNP polymorphism (73.673213 $\mathrm{Mb}$ ) (Figure 6). We then compared alcohol preference of BXDs with the "T" and "G" genotypes. Although not reaching a significant level, the average alcohol consumption of the " $G$ " genotype strains was higher than that of the " $T$ " genotype strains (Figure 7). 
A

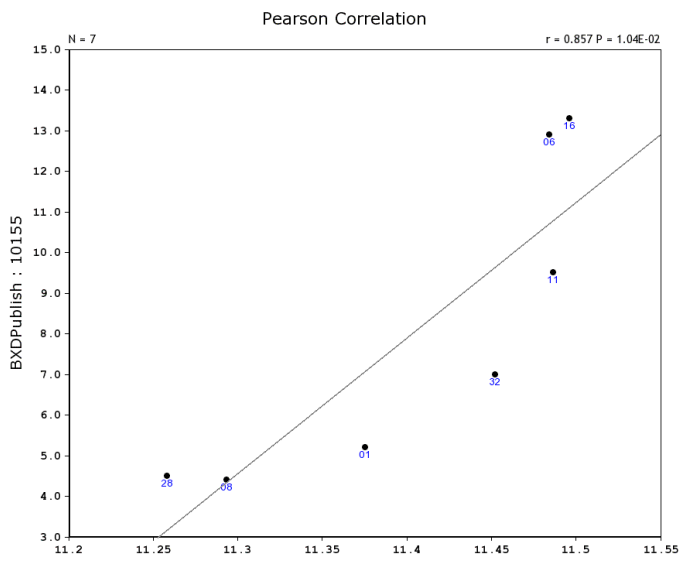

UCHSC BXD Whole Brain M430 2.0 (Nov06) RMA : 1452116_s_at

B

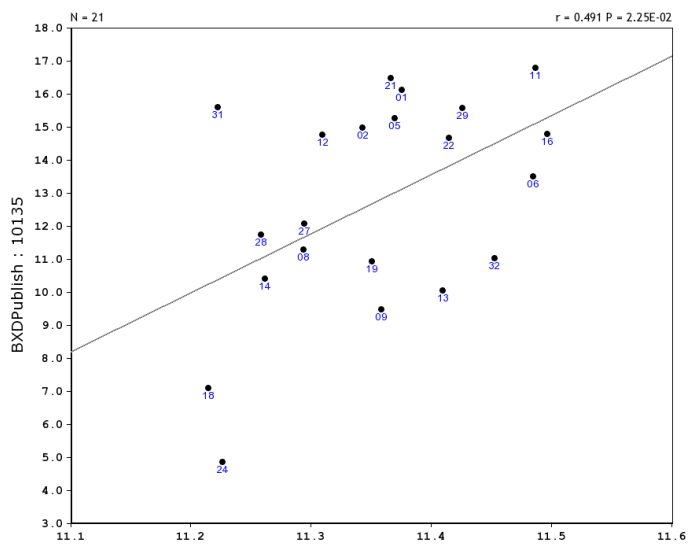

C

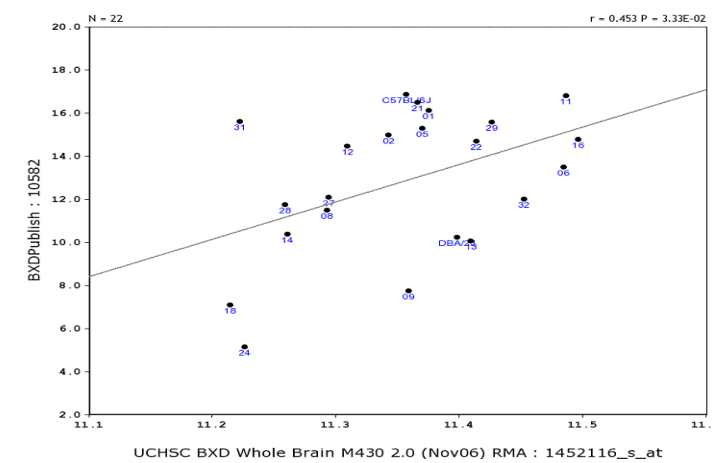

Figure 4. Expression level of Atf2 in whole brain was correlated to the alcohol consumption. Y axis indicates the alcohol consumption $(\mathrm{g} / \mathrm{kg})$ while $\mathrm{X}$ is the relative strength of the signal of the gene expression. A. Expression of Atf2 probe 1452116 is positively correlated to for average amount of ethanol consumption during 3-week free choice period using two-bottle choice test for males. B. Expression of Atf 2 probe 1452116 is positively correlated to average amount of ethanol consumed corrected for body weight for a 2-week. C. Expression was positively related to total consumption over $24 \mathrm{~h}$ for males $(\mathrm{g} / \mathrm{kg})$. 


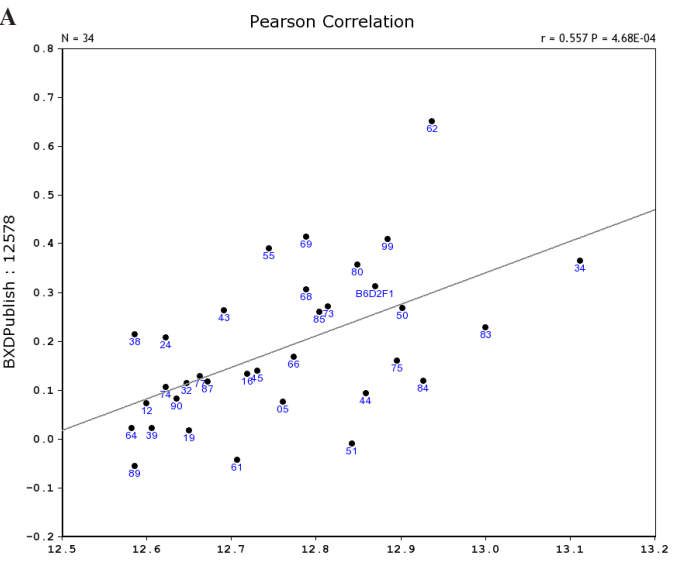

B

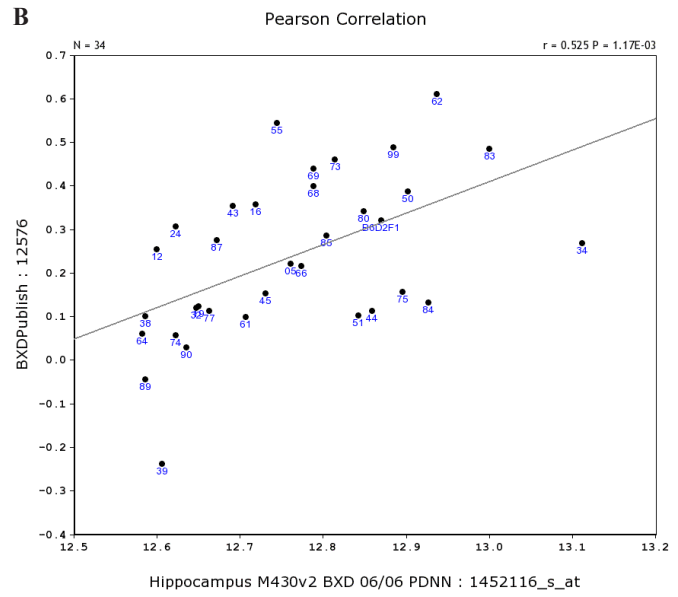

C

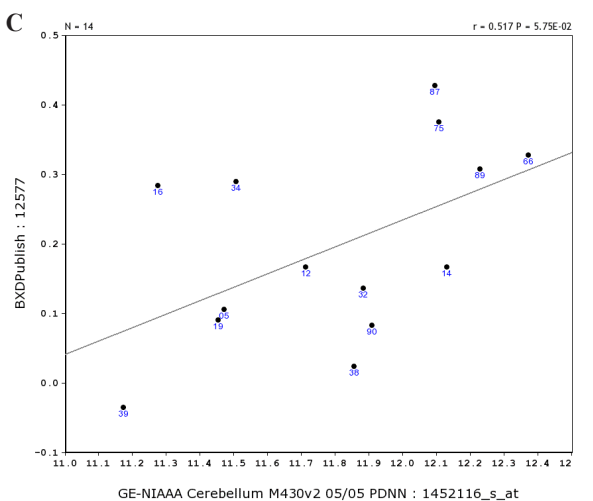

D

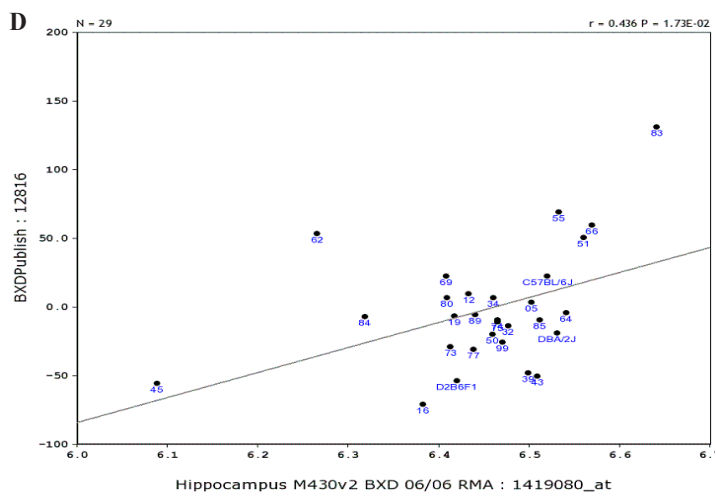

Figure 5. Co-expression of Atf 2 in hippocampus and alcohol preference in hippocampus. Y axis indicates the alcohol consumption $(\mathrm{g} / \mathrm{kg})$ while $\mathrm{X}$ is the relative strength of the signal of the gene expression. A. Probe 1452116 of Atf 2 was positively correlated with ethanol average consumption of BXD strains at 2 weeks from a study using twobottle choice and 2-h access. B. Probe 1452116 of Atf 2 was positively correlated with ethanol average consumption of BXD strains at 4 weeks from a study using two-bottle choice and 2-h access. C. Expression of Atf2 (Probe 1452116) positively correlated to the amount of average ethanol consumption in week 3 for BXD strains using a two-bottle choice, 2-h access. D. Expression of Atf2 was also correlated to the ethanol consumption from data of 3 days after cycle 1 of chronic intermittent ethanol vapor in a recently study using 2-bottle choice consumption.

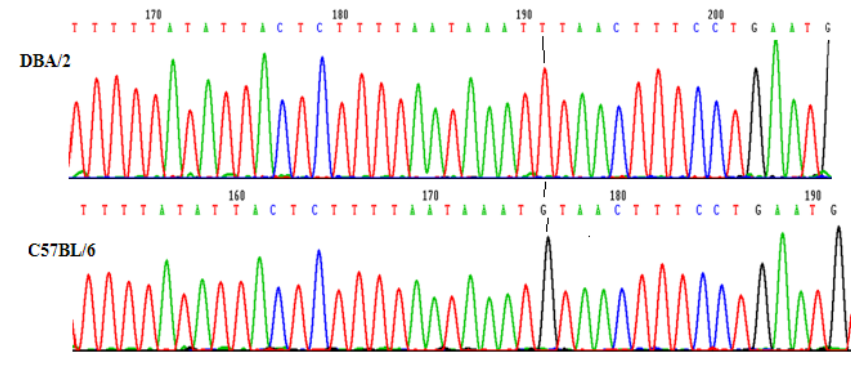

Figure 6. Re-sequence for the confirmation of the T/G SNP of Atf2 at position Chr2:73673213 between the C57BL/6 and DBA/2 strains. Vertical black bar indicates the polymorphism between B6 and D2 in the Atf 2 . 


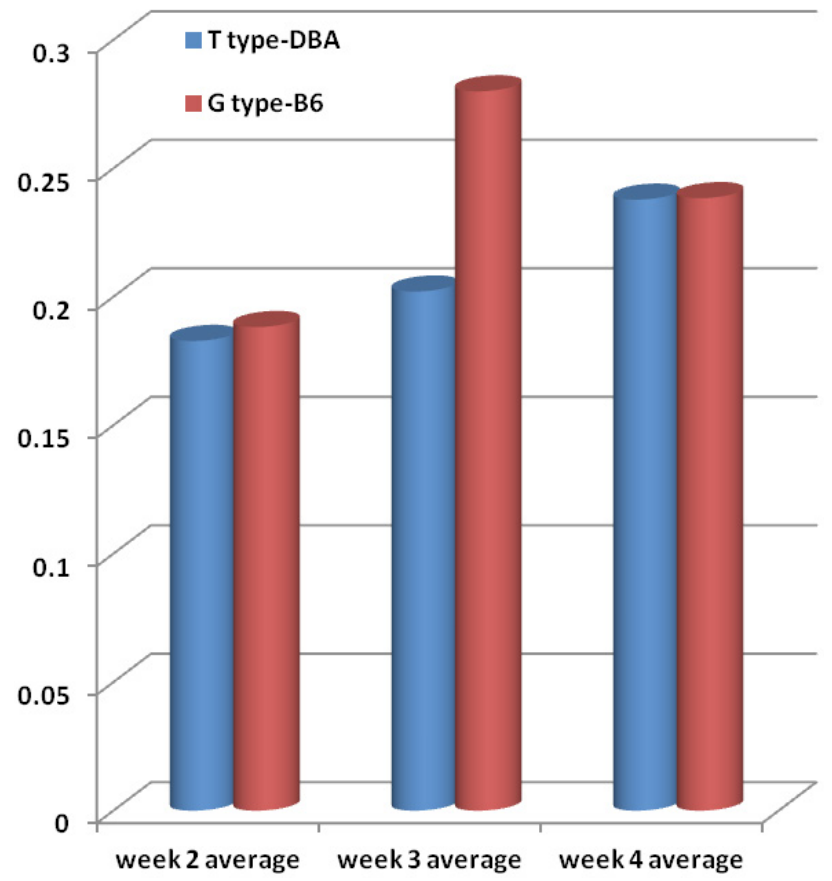

Figure 7. Alcohol preference of BXD strains of " $T$ " genotype and "G" genotype. The average alcohol consumptions for weeks 2, 3, and 4 of BXD strains of the " $G$ " genotype strains were higher than that of the " $T$ " genotype strains.

\section{SNP genotypes of $A T F 2$ and alcohol dependence in humans}

Association analyses were carried out using the SNP genotypes of the ATF2 gene and flanking regions. The results showed evidence of weak associations between rs 13006117 in the 5 ' upstream region and rs7567205 in the $3^{\prime}$ upstream region of the ATF2 gene and alcohol dependence in African Americans ( $\mathrm{P}=0.035$ and 0.052 , respectively) (Table 2).

Table 2. Case-control association analysis between the $A T F 2$ gene and alcohol dependence in the African and European Americans.

\begin{tabular}{|c|c|c|c|c|c|c|c|c|c|c|c|}
\hline \multirow[t]{2}{*}{ SNPs } & \multicolumn{6}{|c|}{ African/American } & \multicolumn{3}{|c|}{ European/American } & \multicolumn{2}{|c|}{ Meta-analysis } \\
\hline & Positions & Regions & Alleles & Cases & Controls & $\mathrm{P}$ & Cases & Controls & $\mathrm{P}$ & OR & $\mathrm{P}(\mathrm{T})$ \\
\hline rs2358891 & 175558804 & 3'-upstream & $\mathrm{A} / \mathrm{G}$ & 0.274 & 0.3 & 0.048 & 0.234 & 0.292 & 0.0056 & $0.84(0.75-0.94)$ & $0.0018 *$ \\
\hline rs7567205 & 175585832 & 3'-upstream & $\mathrm{G} / \mathrm{A}$ & 0.379 & 0.352 & 0.035 & 0.374 & 0.385 & 0.8 & $1.11(1-1.23)$ & 0.0499 \\
\hline rs1153672 & 175695219 & Intron & $\mathrm{A} / \mathrm{G}$ & 0.136 & 0.122 & 0.11 & 0.093 & 0.066 & 0.31 & $1.14(0.98-1.33)$ & 0.079 \\
\hline rs 13388308 & 175714273 & Intron & $\mathrm{A} / \mathrm{G}$ & 0.452 & 0.427 & 0.052 & 0.203 & 0.205 & 0.95 & $1.1(1-1.22)$ & 0.062 \\
\hline rs7603533 & 175718586 & Intron & $\mathrm{A} / \mathrm{G}$ & 0.14 & 0.126 & 0.11 & 0.095 & 0.066 & 0.27 & $1.14(0.99-1.33)$ & 0.076 \\
\hline rs 13006117 & 175745728 & 5'-upstream & $\mathrm{A} / \mathrm{G}$ & 0.144 & 0.127 & 0.054 & 0.102 & 0.075 & 0.33 & $1.17(1.01-1.35)$ & 0.037 \\
\hline rs 13030474 & 175759749 & 5'-upstream & $\mathrm{A} / \mathrm{C}$ & 0.166 & 0.15 & 0.11 & 0.141 & 0.115 & 0.41 & $1.13(0.99-1.3)$ & 0.077 \\
\hline rs374954 & 175793036 & 5'-upstream & $\mathrm{C} / \mathrm{A}$ & 0.416 & 0.394 & 0.095 & 0.253 & 0.23 & 0.56 & $1.1(0.99-1.21)$ & 0.078 \\
\hline
\end{tabular}

${ }^{a}$ The cases are individuals diagnosed with alcohol dependence. For rs2358891, the controls are the individuals without alcohol dependence. For all the other SNPs, the controls are individuals without alcohol, cocaine, marijuana, opiate, tobacco, stimulant, sedative or other drug dependence. Other results are not shown. *The $P(\mathrm{Q})$ was 0.18 and the fixed effects model was applied. Only the selected SNPs with point-wise marginal association $(\mathrm{P}<0.1)$ are shown in this Table. 
The $\mathrm{P}$ values of the combined (by meta-analysis) African and European American samples were 0.037 and 0.0499 and the odds ratio was 1.17 (1.01-1.35) and 1.11 (1-1.23), respectively. Furthermore, based on the imputted genotypes, rs2358891, in the 3 ' upstream, revealed evidence of significant association in both African Americans and European Americans with P values of 0.048 and 0.0056 , respectively. The meta-analysis of the two populations showed a P value of 0.0018 and odds ratio of 0.84 (0.75-0.94). However, none of the SNPs remained significant after correction for multiple testing even when LD structure of the SNPs was taken into account. Detailed results for these selected SNPs with point-wise marginal association are shown in Table 2.

\section{DISCUSSION}

Our data demonstrated that Atf2 is potentially the causal gene for the QTL on Chr2 that regulates alcohol preference tested using a two-bottle choice in mice. An integrative approach using expression data, SNP segregation, and multiple strain comparisons allowed us to narrow the candidate genes in the QTL region to Atf2. The molecular mechanism of Atf2 in regulating alcohol preference is unclear. One likely mechanism is through the MARK pathway. Atf 2 is known to be involved in the MAPK (MEKK1/MKK7/JNK2) pathway, which has been shown to be altered in alcoholism (Chen et al., 1998; Roberto et al., 2003). Atf2 is a transcriptional activator that binds to the cAMP-responsive element $(\mathrm{Cre})$. Interaction with Jun redirects Jun to bind to Cres preferentially over the 12-O-tetradecanoylphorbol13-acetate response elements (Tres) as part of an Atf2-c-Jun complex (487 aa). However, human study did not show strong support for the association between ATF2 and alcoholism. Alcoholism is particularly influenced by the environment. None of the correlations between expression level and genotypes of Atf 2 and alcohol preference in BXD mice or humans was highly significant. The weak association probably reflects the small contribution of the QTL on $\mathrm{Chr} 2$ to alcohol preference.

We should note that $S p 3$ and $S p 9$ are both trans-acting transcription factors that recognize target sequences and interact with other transcription factors to regulate the expression of other genes. Because their regulatory functions have not been clearly defined, they cannot be ruled out as candidates.

\section{ACKNOWLEDGMENTS}

Research supported by the National Institutes of Health, United States (research grants \#AA016342, \#DA12849, \#DA12690, \#AA017535, \#AA12870, and \#AA11330). Genotyping services were provided by the Center for Inherited Disease Research (CIDR). CIDR is fully funded through a federal contract from the National Institutes of Health to The Johns Hopkins University (contract \#N01-HG-65403). Authors thank Dr. Robert Williams for his great assistance on the analysis of data in GeneNetwork and kindly reviewing of the manuscript.

Dr. Kranzler has been a paid consultant for Alkermes, Lilly, Lundbeck, Pfizer, and Roche. He also reports associations with Eli Lilly, Janssen, Schering Plough, Lundbeck, Alkermes, GlaxoSmithKline, Abbott, and Johnson \& Johnson, as these companies provide support to the ACNP Alcohol Clinical Trials Initiative (ACTIVE) and Dr. Kranzler has received honoraria from ACTIVE. The other authors report no financial interests or potential conflicts of interest. 


\section{$\underline{\text { Supplementary material }}$}

\section{REFERENCES}

American Psychiatric Association (1994). Diagnostic and Statistical Manual of Mental Disorders (DSM) Fourth Edition. American Psychiatric Press, Washington.

Bennett B, Beeson M, Gordon L, Carosone-Link P, et al. (2002). Genetic dissection of quantitative trait loci specifying sedative/hypnotic sensitivity to ethanol: mapping with interval-specific congenic recombinant lines. Alcohol. Clin. Exp. Res. 26: 1615-1624.

Chen J, Ishac EJ, Dent P, Kunos G, et al. (1998). Effects of ethanol on mitogen-activated protein kinase and stressactivated protein kinase cascades in normal and regenerating liver. Biochem. J. 334 (Pt 3): 669-676.

Fernandez JR, Vogler GP, Tarantino LM, Vignetti S, et al. (1999). Sex-exclusive quantitative trait loci influences in alcohol-related phenotypes. Am. J. Med. Genet. 88: 647-652.

Fitzgerald DM, Charness ME, Leite-Morris KA and Chen S (2011). Effects of ethanol and NAP on cerebellar expression of the neural cell adhesion molecule L1. PLoS One 6: e24364.

Gelernter J, Panhuysen C, Weiss R, Brady K, et al. (2005). Genomewide linkage scan for cocaine dependence and related traits: significant linkages for a cocaine-related trait and cocaine-induced paranoia. Am. J. Med. Genet. B Neuropsychiatr. Genet. 136B: 45-52.

Gill K, Liu Y and Deitrich RA (1996). Voluntary alcohol consumption in BXD recombinant inbred mice: relationship to alcohol metabolism. Alcohol. Clin. Exp. Res. 20: 185-190.

Grisel JE, Metten P, Wenger CD, Merrill CM, et al. (2002). Mapping of quantitative trait loci underlying ethanol metabolism in BXD recombinant inbred mouse strains. Alcohol. Clin. Exp. Res. 26: 610-616.

Howie BN, Donnelly P and Marchini J (2009). A flexible and accurate genotype imputation method for the next generation of genome-wide association studies. PLoS Genet. 5: e1000529.

Jeffrey SS, Fero MJ, Børresen-Dale AL and Botstein D (2002). Expression array technology in the diagnosis and treatment of breast cancer. Mol. Interv. 2: 101-109.

Jiao Y, Zhang J, Yan J, Stuart J, et al. (2011). Differential gene expression between wild-type and Gulo-deficient mice supplied with vitamin C. Genet. Mol. Biol. 34: 386-395.

Kamens HM, McKinnon CS, Li N, Helms ML, et al. (2009). The alpha 3 subunit gene of the nicotinic acetylcholine receptor is a candidate gene for ethanol stimulation. Genes Brain Behav. 8: 600-609.

Li HS, Zhang JY, Thompson BS, Deng XY, et al. (2001). Rat mitochondrial ATP synthase ATP5G3: cloning and upregulation in pancreas after chronic ethanol feeding. Physiol. Genomics 6: 91-98.

Parsons MJ, Grimm C, Paya-Cano JL, Fernandes C, et al. (2012). Genetic variation in hippocampal microRNA expression differences in C57BL/6 J X DBA/2 J (BXD) recombinant inbred mouse strains. BMC Genomics 13: 476.

Philip VM, Duvvuru S, Gomero B, Ansah TA, et al. (2010). High-throughput behavioral phenotyping in the expanded panel of BXD recombinant inbred strains. Genes Brain Behav. 9: 129-159.

Pierucci-Lagha A, Gelernter J, Chan G, Arias A, et al. (2007). Reliability of DSM-IV diagnostic criteria using the semistructured assessment for drug dependence and alcoholism (SSADDA). Drug. Alcohol Depend. 91: 85-90.

Roberto M, Nelson TE, Ur CL, Brunelli M, et al. (2003). The transient depression of hippocampal CA1 LTP induced by chronic intermittent ethanol exposure is associated with an inhibition of the MAP kinase pathway. Eur. J. Neurosci. 17: 1646-1654.

Rodríguez FD, Simonsson P and Alling C (1992). A method for maintaining constant ethanol concentrations in cell culture media. Alcohol Alcohol. 27: 309-313.

Ruf C, Carosone-Link P, Springett J and Bennett B (2004). Confirmation and genetic dissection of a major quantitative trait locus for alcohol preference drinking. Alcohol. Clin. Exp. Res. 28: 1613-1621.

Sharov AA, Masui S, Sharova LV, Piao Y, et al. (2008). Identification of Pou5f1, Sox2, and Nanog downstream target genes with statistical confidence by applying a novel algorithm to time course microarray and genome-wide chromatin immunoprecipitation data. BMC Genomics 9: 269. doi: 10.1186/1471-2164-9-269.

Xiong Q, Qiu Y and Gu W (2008). PGMapper: a web-based tool linking phenotype to genes. Bioinformatics 24: 1011-1013.

Yan J, Jiao Y, Li X, Jiao F, et al. (2007). Evaluation of gene expression profiling in a mouse model of L-gulonolactone oxidase gene deficiency. Genet. Mol. Biol. 30: 322-329.

Yu T, Lee YJ, Yang HM, Han S, et al. (2011). Inhibitory effect of Sanguisorba officinalis ethanol extract on NO and PGE(2) production is mediated by suppression of NF-kappaB and AP-1 activation signaling cascade. J. Ethnopharmacol. 134: 11-17. 